\title{
Time-to-depth conversion and seismic velocity estimation using time-migration velocity ${ }^{a}$
}

${ }^{a}$ Published in Geophysics, 73, no. 5, VE205-VE210, (2008)

Maria Cameron*, Sergey Fomel ${ }^{\dagger}$, and James Sethian ${ }^{\ddagger}$

\begin{abstract}
The objective of this work is to build an efficient algorithm (a) to estimate seismic velocity from time-migration velocity, and (b) to convert time-migrated images to depth. We establish theoretical relations between the time-migration velocity and the seismic velocity in 2-D and 3-D using paraxial ray tracing theory. The relation in 2-D implies that the conventional Dix velocity is the ratio of the interval seismic velocity and the geometrical spreading of the image rays. We formulate an inverse problem of finding seismic velocity from the Dix velocity and develop a numerical procedure for solving it. This procedure consists of two steps: (1) computation of the geometrical spreading of the image rays and the true seismic velocity in the time-domain coordinates from the Dix velocity; (2) conversion of the true seismic velocity from the time domain to the depth domain and computation of the transition matrices from time-domain coordinates to depth.

For step 1, we derive a partial differential equation (PDE) in 2-D and 3-D relating the Dix velocity and the geometrical spreading of the image rays to be found. This is a nonlinear elliptic PDE. The physical setting allows us to pose a Cauchy problem for it. This problem is ill-posed. However we are able to solve it numerically in two ways on the required interval of time. One way is a finite difference scheme inspired by the Lax-Friedrichs method. The second way is a spectral Chebyshev method. For step 2, we develop an efficient Dijkstra-like solver motivated by Sethian's Fast Marching Method.

We test our numerical procedures on a synthetic data example and apply them to a field data example. We demonstrate that our algorithms give significantly more accurate estimate of the seismic velocity than the conventional Dix inversion. Our velocity estimate can be used as a reasonable first guess in building velocity models for depth imaging.
\end{abstract}




\section{INTRODUCTION}

Time-domain seismic imaging is a robust and efficient process routinely applied to seismic data (Yilmaz, 2001; Robein, 2003). Rapid scanning and determination of time-migration velocity can be accomplished either by repeated migrations (Yilmaz et al., 2001) or by velocity continuation (Fomel, 2003). Time migration is considered adequate for seismic imaging in areas with mild lateral velocity variations. However, even mild variations can cause structural distortions of time-migrated images and render them inadequate for accurate geological interpretation of subsurface structures.

To remove structural errors inherent in time migration, it is necessary to convert time-migrated images into the depth domain either by migrating the original data with a prestack depth migration algorithm or by depth migrating post-stack data after time demigration (Kim et al., 1997). Each of these options requires converting the time migration velocity to a velocity model in depth.

The connection between the time- and depth-domain coordinates is provided by the concept of image ray, introduced by Hubral (1977). Image rays are seismic rays that arrive normal to the Earth's surface. Hubral's theory explains how a depth velocity model can be converted to the time coordinates. However, it does not explain how a depth velocity model can be converted to the time-migration velocity. Moreover, image-ray tracing is a numerically inconvenient procedure for achieving uniform coverage of the subsurface. This may explain why simplified image-ray-tracing algorithms (Larner et al., 1981; Hatton et al., 1981) did not find widespread application in practice. Other limitations of image rays are related to the inability of time migration to handle large lateral variations in velocity (Bevc et al., 1995; Robein, 2003).

The objective of the present work is to find an efficient method for building a velocity model from time-migration velocity. We establish new ray-theoretic connections between time-migration velocity and seismic velocity in $2-\mathrm{D}$ and $3-\mathrm{D}$. These results are based on the image ray theory and the paraxial ray tracing theory (Popov and Pšenčik, 1978; Červený, 2001; Popov, 2002). Our results can be viewed as an extension of the Dix formula (Dix, 1955) to laterally inhomogeneous media. We show that the Dix velocity is seismic velocity divided by the geometrical spreading of the image rays. Hence, we use the Dix velocity instead of time migration velocity as a more convenient input. We develop a numerical approach to find (a) seismic velocity from the Dix velocity, and (b) transition matrices from the time-domain coordinates to the depth-domain coordinates. We test our approach on synthetic and field data examples.

Our approach is complementary to more traditional velocity estimation methods and can be used as the first step in a velocity model building process. 


\section{TIME MIGRATION VELOCITY}

Kirchhoff prestack time migration is commonly based on the following travel time approximation (Yilmaz, 2001). Let $\mathbf{s}$ be a source, $\mathbf{r}$ be a receiver, and $\mathbf{x}$ be the reflection subsurface point. Then the total travel time from $\mathbf{s}$ to $\mathbf{x}$ and from $\mathbf{x}$ to $\mathbf{r}$ is approximated as

$$
T(\mathbf{s}, \mathbf{x})+T(\mathbf{x}, \mathbf{r}) \approx \hat{T}\left(\mathbf{x}_{0}, t_{0}, \mathbf{s}, \mathbf{r}\right)
$$

where $\mathbf{x}_{0}$ and $t_{0}$ are effective parameters of the subsurface point $\mathbf{x}$. The approximation $\hat{T}$ usually takes the form of the double-square-root equation

$$
\hat{T}\left(\mathbf{x}_{0}, t_{0}, \mathbf{s}, \mathbf{r}\right)=\sqrt{t_{0}^{2}+\frac{\left|\mathbf{x}_{0}-\mathbf{s}\right|^{2}}{v_{m}^{2}\left(\mathbf{x}_{0}, t_{0}\right)}}+\sqrt{t_{0}^{2}+\frac{\left|\mathbf{x}_{0}-\mathbf{r}\right|^{2}}{v_{m}^{2}\left(\mathbf{x}_{0}, t_{0}\right)}},
$$

where $\mathbf{x}_{0}$ and $t_{0}$ are the escape location and the travel time of the image ray (Hubral, 1977) from the subsurface point $\mathbf{x}$. Regarding this approximation, let us list four cases depending on the seismic velocity $v$ and the dimension of the problem:

2-D and $3-\mathbf{D}$, velocity $v$ is constant. Equation 2 is exact, and $v_{m}=v$.

2-D and 3-D, velocity $v$ depends only on the depth $z$. Equation 2 is a consequence of the truncated Taylor expansion for the travel time around the surface point $\mathbf{x}_{0}$. Velocity $v_{m}$ depends only on $t_{0}$ and is the root-mean-square velocity:

$$
v_{m}\left(t_{0}\right)=\sqrt{\frac{1}{t_{0}} \int_{0}^{t_{0}} v^{2}(z(t)) d t} .
$$

In this case, the Dix inversion formula (Dix, 1955) is exact. We formally define the Dix velocity $v_{\text {Dix }}(t)$ by inverting equation 3 , as follows:

$$
v_{D i x}(t)=\sqrt{\frac{d}{d t_{0}}\left(t_{0} v_{m}^{2}\left(t_{0}\right)\right)} .
$$

2-D, velocity is arbitrary. Equation 2 is a consequence of the truncated Taylor expansion for the travel time around the surface point $x_{0}$. Velocity $v_{m}\left(x_{0}, t_{0}\right)$ is a certain kind of mean velocity, and we establish its exact meaning in the next section.

3-D, velocity is arbitrary. Equation 2 is heuristic and is not a consequence of the truncated Taylor expansion. In order to write an analog of travel time approximation 2 for 3-D, we use the relation (Hubral and Krey, 1980)

$$
\boldsymbol{\Gamma}=\left[v\left(\mathbf{x}_{0}\right) \mathbf{R}\left(\mathbf{x}_{0}, t_{0}\right)\right]^{-1},
$$

where $\boldsymbol{\Gamma}$ is the matrix of the second derivatives of the travel times from a subsurface point $\mathbf{x}$ to the surface, $\mathbf{R}$ is the matrix of radii of curvature of the emerging wave front from the point source $\mathbf{x}$, and $v\left(\mathbf{x}_{0}\right)$ is the velocity at the 
surface point $\mathbf{x}_{0}$. For convenience, we prefer to deal with matrix $\mathbf{K} \equiv \boldsymbol{\Gamma}^{-1}$, which, according to equation 5 is

$$
\mathbf{K}\left(\mathbf{x}_{0}, t_{0}\right) \equiv v\left(\mathbf{x}_{0}\right) \mathbf{R}\left(\mathbf{x}_{0}, t_{0}\right)
$$

The travel time approximation for 3-D implied by the Taylor expansion is

$$
\begin{gathered}
\hat{T}\left(\mathbf{x}_{0}, t_{0}, \mathbf{s}, \mathbf{r}\right) \\
=\sqrt{t_{0}^{2}+t_{0}\left(\mathbf{x}_{0}-\mathbf{s}\right)^{T}\left[\mathbf{K}\left(\mathbf{x}_{0}, t_{0}\right)\right]^{-1}\left(\mathbf{x}_{0}-\mathbf{s}\right)} \\
+\sqrt{t_{0}^{2}+t_{0}\left(\mathbf{x}_{0}-\mathbf{r}\right)^{T}\left[\mathbf{K}\left(\mathbf{x}_{0}, t_{0}\right)\right]^{-1}\left(\mathbf{x}_{0}-\mathbf{r}\right)} .
\end{gathered}
$$

The entries of the matrix $\frac{1}{t_{0}} \mathbf{K}\left(\mathbf{x}_{0}, t_{0}\right)$ have dimension of squared velocity and can be chosen optimally in the process of time migration. It is possible to show, however, that one needs only the values of

$$
\operatorname{det}\left(\frac{\partial}{\partial t_{0}} \mathbf{K}\left(\mathbf{x}_{0}, t_{0}\right)\right)
$$

to perform the inversion. This means that the conventional 3-D prestack time migration with traveltime approximation 2 provides sufficient input for our inversion procedure in 3-D. The determinant in equation 8 is well approximated by the square of the Dix velocity obtained from the 3-D prestack time migration using the approximation given by equation 2 .

One can employ more complex and accurate approximations than the double-squareroot equations 2 and 7, i.e. the shifted hyperbola approximation (Siliqi and Bousquié, 2000). However, other known approximations also involve parameters equivalent to $v_{m}$ or $\mathbf{K}$.

\section{SEISMIC VELOCITY}

In this section, we will establish theoretical relationships between time-migration velocity and seismic velocity in 2-D and 3-D.

The seismic velocity and the Dix velocity are connected through the quantity $\mathbf{Q}$, the geometrical spreading of image rays. $\mathbf{Q}$ is a scalar in $2-\mathrm{D}$ and a $2 \times 2$ matrix in 3 -D. The simplest way to introduce $\mathbf{Q}$ is the following. Trace an image ray $\mathbf{x}\left(\mathbf{x}_{0}, t\right)$. $\mathbf{x}_{0}$ is the starting surface point, $t$ is the traveltime. Call this ray central. Consider a small tube of rays around it. All these rays start from a small neighborhood $d \mathbf{x}_{0}$ of the point $\mathbf{x}_{0}$ perpendicular to the earth surface. Thus, they represent a fragment of a plane wave propagating downward. Consider the fragment of the wave front defined by this ray tube at time $t_{0}$. Let $d \mathbf{q}$ be the fragment of the tangent to the front at the point $\mathbf{x}\left(\mathbf{x}_{0}, t_{0}\right)$ reached by the central ray at time $t_{0}$, bounded by the ray tube (Figure 1 ). Then, in $2-\mathrm{D}, Q$ is the derivative $Q\left(x_{0}, t_{0}\right)=\frac{d q}{d x_{0}}$. In $3-\mathrm{D}, \mathbf{Q}$ is the matrix of the derivatives $\mathbf{Q}_{i j}\left(\mathbf{x}_{0}, t_{0}\right)=\frac{d \mathbf{q}_{i}}{d \mathbf{x}_{0 j}}, i, j=1,2$, where derivatives are 


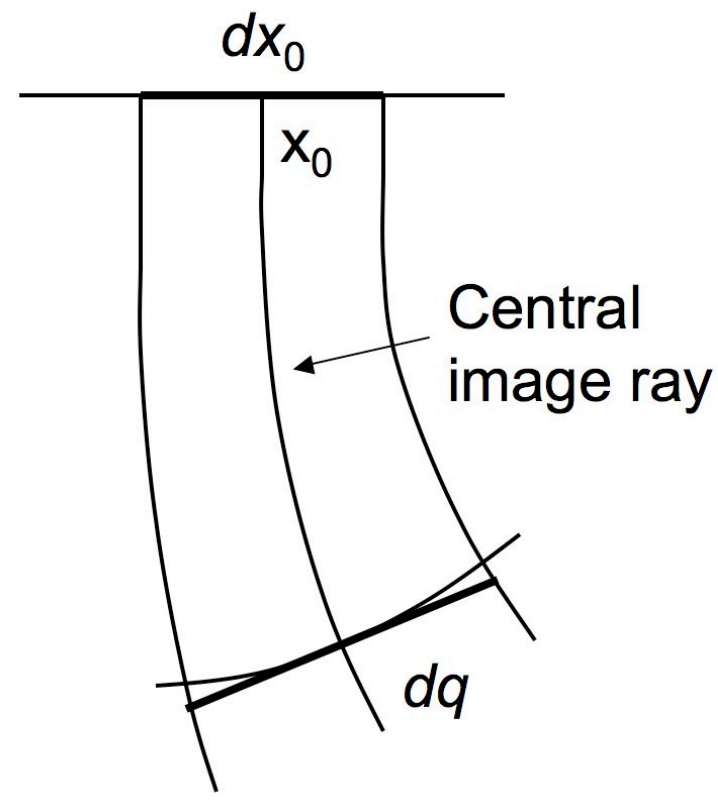

Figure 1: Illustration for the definition of geometrical spreading.

taken along certain mutually orthogonal directions $\mathbf{e}_{1}, \mathbf{e}_{2}$ (Popov and Pšenčik, 1978; Červený, 2001; Popov, 2002).

The time evolution of the matrices $\mathbf{Q}$ and $\mathbf{P}$ is given by

$$
\frac{d}{d t}\left(\begin{array}{l}
\mathbf{Q} \\
\mathbf{P}
\end{array}\right)=\left(\begin{array}{cc}
\mathbf{0} & v_{0}^{2} \mathbf{I} \\
-\frac{1}{v_{0}} \mathbf{V} & \mathbf{0}
\end{array}\right)\left(\begin{array}{c}
\mathbf{Q} \\
\mathbf{P}
\end{array}\right),
$$

where $v_{0}$ it the velocity at the central ray at time $t, \mathbf{V}=\left(\frac{\partial^{2} v}{\partial q_{i} \partial q_{j}}\right)_{i, j=1,2}$, and $\mathbf{I}$ is the $2 \times 2$ identity matrix. The absolute value of $\operatorname{det} \mathbf{Q}$ has a simple meaning: it is the geometrical spreading of the image rays (Popov and Pšenčik, 1978; Červený, 2001; Popov, 2002). The matrix $\boldsymbol{\Gamma}$, introduced in the previous section, relates to $\mathbf{Q}$ and $\mathbf{P}$ as $\boldsymbol{\Gamma}=\mathbf{P Q}^{-1}$. Hence, $\mathbf{K}=\mathbf{Q P}^{-1}$.

In (Cameron et al., 2007), we have proven that

$$
v_{D i x}\left(x_{0}, t_{0}\right) \equiv \sqrt{\frac{\partial}{\partial t_{0}}\left(t_{0} v_{m}^{2}\left(x_{0}, t_{0}\right)\right)}=\frac{v\left(x\left(x_{0}, t_{0}\right), z\left(x_{0}, t_{0}\right)\right)}{\left|Q\left(x_{0}, t_{0}\right)\right|}
$$

in 2-D, where $v_{m}\left(x_{0}, t_{0}\right)$ is the time-migration velocity, and

$$
\frac{\partial}{\partial t_{0}}\left(\mathbf{K}\left(\mathbf{x}_{0}, t_{0}\right)\right)=v\left(\mathbf{x}\left(\mathbf{x}_{0}, t_{0}\right)\right)\left(\mathbf{Q}\left(\mathbf{x}_{0}, t_{0}\right) \mathbf{Q}^{T}\left(\mathbf{x}_{0}, t_{0}\right)\right)^{-1}
$$

in 3-D, $\mathbf{K}$ is defined by equation 6 and can be determined from equation 7 . 


\section{PARTIAL DIFFERENTIAL EQUATIONS FOR THE GEOMETRICAL SPREADING OF IMAGE RAYS}

In this section, we derive the partial differential equations for $Q$ in 2-D and 3-D. From now on, we will denote the square of the Dix velocity by $f$ in $2-\mathrm{D}$ and the corresponding matrix by $\mathbf{F}$ in $3-\mathrm{D}$, to avoid the subscript:

$$
\mathbf{F} \equiv \frac{\partial}{\partial t_{0}}\left(\mathbf{K}\left(\mathbf{x}_{0}, t_{0}\right)\right)
$$

Furthermore, we imply that $t_{0}$ denotes the one-way traveltime along the image rays. Finally, we assume that our domain does not contain caustics, i.e., the image rays do not cross on the interval of time we consider.

\section{2-D case}

Consider a set of image rays coming to the surface. Suppose we are tracing them all backwards in time together with the quantities $Q$ and $P$. Let us eliminate the unknown velocity $v$ in system 9 using equation 10. Moreover, let us eliminate the differentiation in $q$ using the definition of $Q$ and rewrite it in the time-domain coordinates $\left.x_{0}, t_{0}\right)$. Indeed, $Q=\frac{d q}{d x_{0}}$, hence $\frac{d}{d q}=\frac{d}{d x_{0}} \frac{d x_{0}}{d q}=Q^{-1} \frac{d}{d x_{0}}$. Therefore, system 9 becomes

$$
Q_{t_{0}}=(f Q)^{2} P, \quad P_{t_{0}}=-\frac{1}{f Q}\left(\frac{(f Q)_{x_{0}}}{Q}\right)_{x_{0}} \text {. }
$$

Eliminating $P$ in system 13 , we get the following partial differential equation (PDE) for $Q$

$$
\left(\frac{Q_{t_{0}}}{f^{2} Q^{2}}\right)_{t_{0}}=-\frac{1}{f Q}\left(\frac{(f Q)_{x_{0}}}{Q}\right)_{x_{0}} .
$$

The initial conditions are $Q\left(x_{0}, 0\right)=1, Q_{t_{0}}\left(x_{0}, 0\right)=0$. Equation 14 simplifies in terms of the negative reciprocal of $Q$. Introduce $y=-\frac{1}{Q}$. Then equation 14 becomes

$$
\left(\frac{y_{t_{0}}}{f^{2}}\right)_{t_{0}}=\frac{y}{f}\left(\left(\frac{f}{y}\right)_{x_{0}} y\right)_{x_{0}} .
$$

In the expanded form, equation 15 is

$$
\frac{y_{t_{0} t_{0}}}{f^{2}}-2 \frac{y_{t_{0}} f_{t_{0}}}{f^{3}}=y \frac{f_{x_{0} x_{0}}}{f}-y_{x_{0}} \frac{f_{x_{0}}}{f}-y_{x_{0} x_{0}}+\frac{y_{x_{0}}^{2}}{y} .
$$

\section{3-D case}

Equation 11 can be rewritten in the following form

$$
v=\sqrt[4]{\operatorname{det} \mathbf{F}(\operatorname{det} \mathbf{Q})^{2}},
$$


where $\mathbf{F}$ is the left-hand side of equation 11 . As in $2-\mathrm{D}$, we rewrite system 9 in the time-domain coordinates $\left(\mathbf{x}_{0}, t_{0}\right)$. Then we get

$$
\begin{aligned}
\mathbf{Q}_{t_{0}} & =v^{2} \mathbf{P} \\
\mathbf{P}_{t_{0}} & =-\frac{1}{v} \mathbf{Q}^{-1}\left[\nabla\left(\mathbf{Q}^{-1} \nabla v\right)^{T}\right] \mathbf{Q},
\end{aligned}
$$

where $v$ is given by equation 17 , and the gradients are taken with respect to $\mathbf{x}_{0}$. Then the $\mathrm{PDE}$ for $\mathrm{Q}$ is

$$
\left(\frac{1}{v^{2}} \mathbf{Q}_{t_{0}}\right)_{t_{0}}=-\frac{1}{v} \mathbf{Q}^{-1}\left[\nabla\left(\mathbf{Q}^{-1} \nabla v\right)^{T}\right] \mathbf{Q} .
$$

The initial conditions are $\mathbf{Q}\left(\mathbf{x}_{0}, 0\right)=\mathbf{I}_{2}, \mathbf{Q}_{t_{0}}\left(\mathbf{x}_{0}, 0\right)=\mathbf{0}$. The required input $\sqrt{\operatorname{det} \mathbf{F}}$ is well-approximated by the squares of the Dix velocity obtained from the 3 -D prestack time migration. We emphasize that despite the fact that $\mathbf{Q}$ is a matrix in $3-\mathrm{D}$, scalar data are enough for its computation.

\section{Cauchy problem for elliptic equations}

Equations 14 and 20 reveal the nature of the instabilities in the problem in hand. These PDEs are elliptic, while the physical setting allows us to pose only a Cauchy problem for them, which is well-known to be ill-posed. Furthermore, the fact that the PDEs involve not only the Dix velocity itself, but also its first and second derivatives, leads to high sensitivity of the solutions to input data.

Nonetheless, we found two ways for solving these PDEs numerically on the required, and relatively short, interval of time: namely, a finite difference scheme inspired by the Lax-Friedrichs method and a spectral Chebyshev method. A detailed analysis of the problem shows that our methods work thanks to

1. the special input $v_{\text {Dix }}$, corresponding to a positive finite seismic velocity;

2. the special initial conditions $Q\left(x_{0}, t_{0}=0\right)=1, Q_{t}\left(x_{0}, t_{0}=0\right)=0$ corresponding to the image rays;

3. the fact that our finite difference method contains error terms which damp the high harmonics; truncation of the polynomial series in the spectral Chebyshev method which is similar to truncation of the high harmonics; and

4. the short interval of time, in which we need to compute the solution, so that the growing low harmonics fail to develop significantly.

Items 1 and 2 say that the exact solutions of our PDEs for the hypothetical perfect Dix velocity given by equations 10 and 11 are finite and nonzero. Items 3 and 4 say that the numerical methods take care of the imperfection of the data and computations on a short enough time interval. 


\section{INVERSION METHODS}

Our numerical reconstruction of true seismic velocity $v(\mathbf{x})$ in depth-domain coordinates from the Dix velocity given in the time-domain coordinates $\left(\mathbf{x}_{0}, t_{0}\right)$ consists of two steps:

Step 1. Compute the geometrical spreading of the image rays in the time-domain coordinates from the Dix velocity by solving equation 14 in 2-D and 20 in 3-D. Then find $v\left(\mathbf{x}_{0}, t_{0}\right)$ from equation 10 in $2-\mathrm{D}$ and equation 17 in $3-\mathrm{D}$.

Step 2. Convert the seismic velocity $v\left(\mathbf{x}_{0}, t_{0}\right)$ in the time-domain coordinates to the depth-domain coordinates $\mathbf{x}$ using the time-to-depth conversion algorithm, which was presented by Cameron et al. (2007). It is a fast and robust Dijkstralike solver motivated by the Fast Marching method (Sethian, 1996, 1999).

We performed step 1 in two ways: a finite difference method and a spectral Chebyshev method.

\section{Finite difference method}

This method was inspired by the Lax-Friedrichs method for hyperbolic conservation laws Lax (1954) due to its total variation diminishing property. We use the "LaxFriedrichs averaging" and the wide 5-point stencil in space. The scheme is given by

$$
\begin{aligned}
P_{j}^{n+1} & =\frac{P_{j+1}^{n}+P_{j-1}^{n}}{2}-\frac{\Delta t}{4 \Delta x} \frac{1}{v_{j}^{n}}\left(\frac{v_{j+2}^{n}-v_{j}^{n}}{Q_{j+1}^{n}}-\frac{v_{j}^{n}-v_{j-2}^{n}}{Q_{j-1}^{n}}\right), \\
-\frac{1}{Q_{j}^{n+1}} & =-\frac{1}{Q_{j}^{n}}+\frac{\Delta t}{2}\left(\left(f_{j}^{n}\right)^{2} P_{j}^{n}+\left(f_{j}^{n+1}\right)^{2} P_{j}^{n+1}\right),
\end{aligned}
$$

where $v \equiv f Q$. We impose the following boundary conditions $Q_{0}^{n}=Q_{n x-1}^{n}=1, P_{0}^{n}=$ $P_{n x-1}^{n}=0$ corresponding the straight boundary rays. We set the initial conditions $Q_{j}^{0}=1, P_{j}^{0}=0$ corresponding to the initial conditions for the image rays traced backward: $Q=1, P=0$.

\section{Spectral Chebyshev method}

Alternatively, we solve our PDE in the form given by equation 15 by a spectral Chebyshev method Boyd (2001). Using cubic splines, we define the input data at $N_{\text {coef }}$ Chebyshev points. We compute the Chebyshev coefficients and the coefficients of the derivatives in the right-hand side of equation 15 . Then we use a smaller number $N_{\text {eval }}$ of the coefficients for function evaluation. We need to do such Chebyshev 
differentiation twice. Finally we perform the time step using the stable third-order Adams-Bashforth method Boyd (2001), which is

$$
u^{n+1}=u^{n}+\Delta t\left(\frac{23}{12} F^{n}-\frac{4}{3} F^{n-1}+\frac{5}{12} F^{n-2}\right),
$$

where $F^{n} \equiv F\left(u^{n}, x, t^{n}\right)$ is the right-hand side. In numerical examples, we tried $N_{\text {coef }} \geq 100$ and $N_{\text {eval }} \leq 25$. This method allows larger time steps than the finite difference, and has the adjustable parameter $N_{\text {eval }}$.

For step 2, we use a Dijkstra-like solver introduced in Cameron et al. (2007). It is an efficient time-to-depth conversion algorithm motivated by the Fast Marching Method (Sethian, 1996). The input for this algorithm is $v\left(x_{0}, t_{0}\right)$ and the outputs are the seismic velocity $v(x, z)$ and the transition matrices from time-domain to depthdomain coordinates $x_{0}(x, z)$ and $t_{0}(x, z)$. We solve the eikonal equation with an unknown right-hand side coupled with the orthogonality relation

$$
\left|\nabla t_{0}\right|=\frac{1}{v\left(x_{0}(x, z), t_{0}(x, z)\right)}, \quad \nabla t_{0} \cdot \nabla x_{0}=0
$$

The orthogonality relation means that the image rays are orthogonal to the wavefronts. Such time-to-depth conversion is very fast and produces the outputs directly on the depth-domain grid.

\section{EXAMPLES}

\section{Synthetic data example}

Figure 2(a) shows a synthetic velocity model. The model contains a high velocity anomaly that is asymmetric and decays exponentially. The corresponding Dix velocity mapped from time to depth is shown in Figure 2(b). There is a significant difference between both the value and the shape of the velocity anomaly recovered by the Dix method and the true anomaly. The difference is explained by taking into account geometrical spreading of image rays. Figure 2(c) shows the velocity recovered by our method and the corresponding family of image rays. An analogous 3-D example is provided in (Cameron et al., 2007).

\section{Field data example}

Figure 3, taken from (Fomel, 2003), shows a prestack time migrated image from the North Sea and the corresponding time-migration velocity obtained by velocity continuation. The most prominent feature in the image is a salt body which causes significant lateral variations of velocity. Figure 4 compares the Dix velocity converted to depth with the interval velocity model recovered by our method. As in the synthetic example, there is a significant difference between the two velocity caused by 


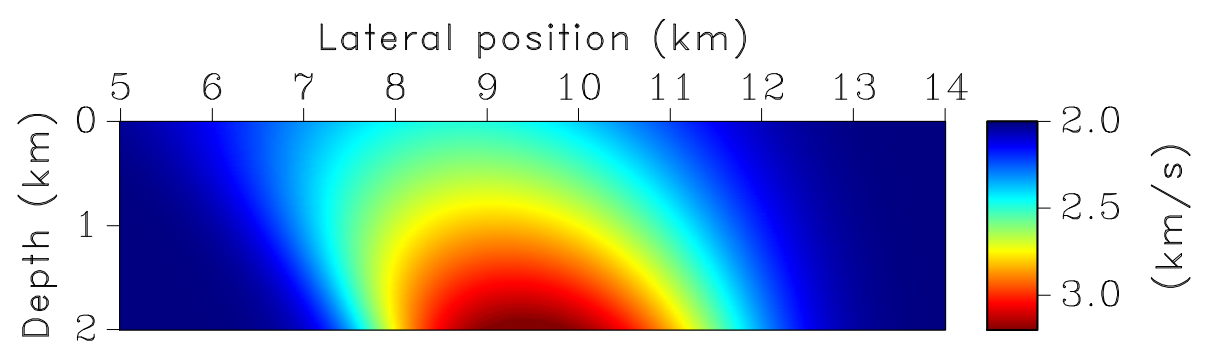

(a)

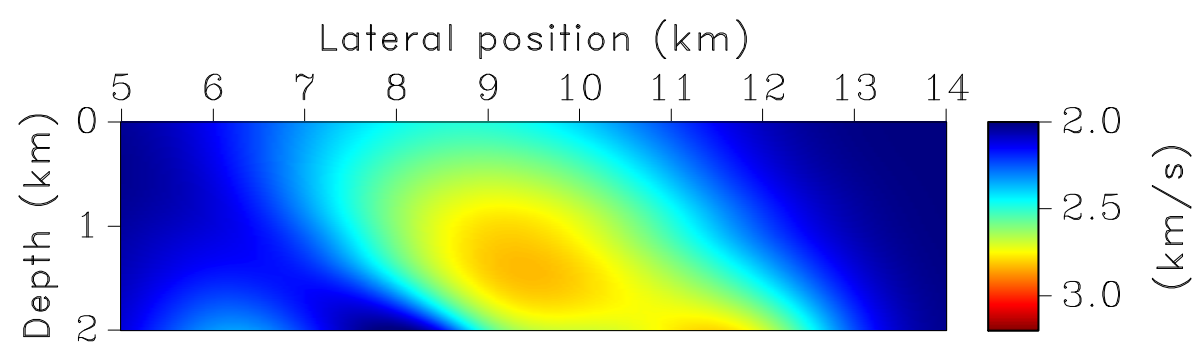

(b)

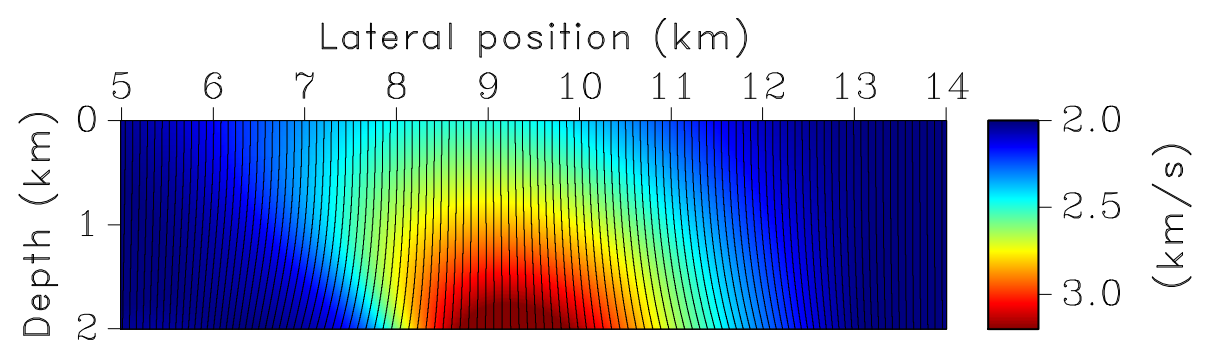

(c)

Figure 2: Synthetic test on interval velocity estimation. (a) Exact velocity model. (b) Dix velocity converted to depth. (c) Estimated velocity model and the corresponding image rays. The image ray spreading causes significant differences between Dix velocity and estimated velocity. 

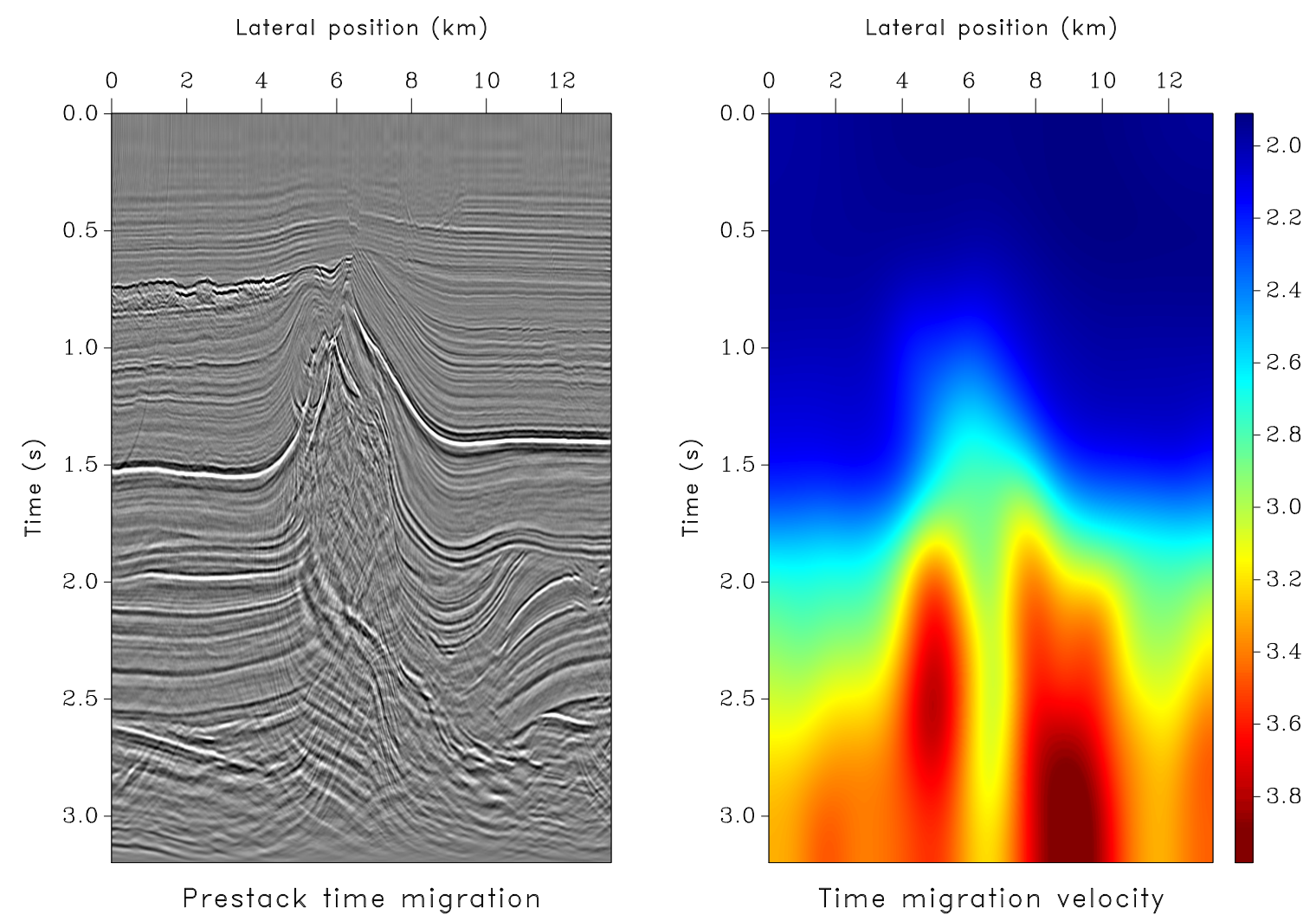

Figure 3: (a) Seismic image from North Sea obtained by prestack time migration using velocity continuation (Fomel, 2003). (b) Corresponding time-migration velocity. 


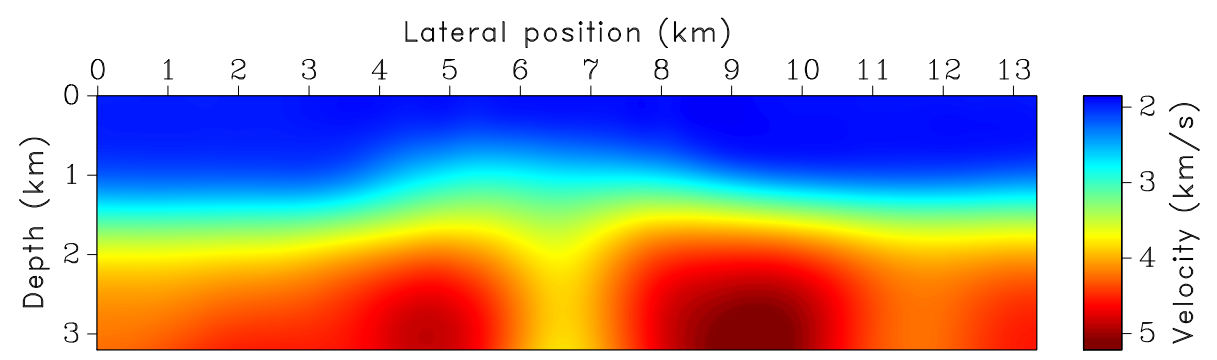

(a)

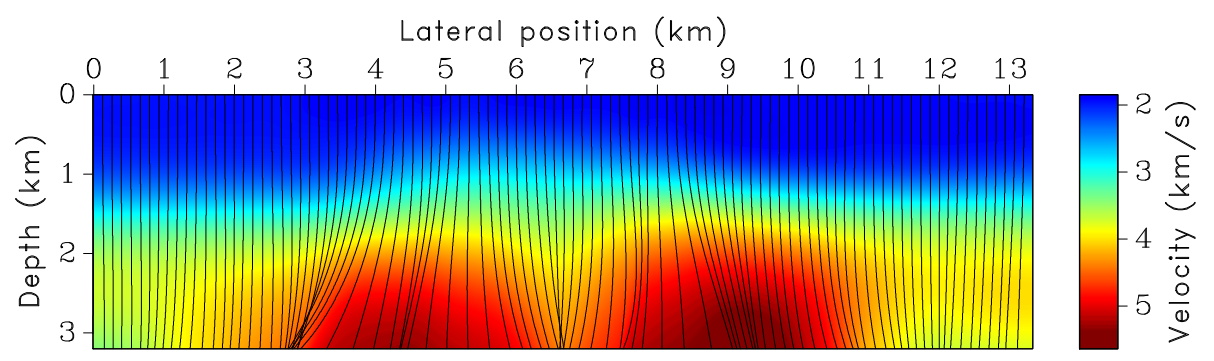

(b)

Figure 4: Field data example of interval velocity estimation. (a) Dix velocity converted to depth. (b) Estimated velocity model and the corresponding image rays. The image-ray spreading causes significant differences between Dix velocity and true velocity.

the geometrical spreading of image rays. The middle part of the velocity model may not be recovered properly. The true structure should include a salt body visible in the image. The inability of our method to recover it exactly shows the limitation of the proposed approach in the areas of significant lateral velocity variations, which invalidate the assumptions behind time migration (Robein, 2003). Figure 5 compares three images: post-stack depth-migration image using Dix velocity, post-stack depth-migration image using the velocity estimated by our method, and prestack time-migration image converted to depth with our algorithm. The evident structural improvements in Figure 5(b) in comparison with Figure 5(a), in particular near salt flanks, and a good structural agreement between Figures 5(b) and 5(c) serve as an indirect evidence of the algorithm success. An ultimate validation should come from prestack depth migration velocity analysis, which is significantly more expensive.

\section{CONCLUSIONS}

We have applied the recently established theorem that the Dix velocity obtainable from the time-migration velocity is the true interval velocity divided by the geometrical spreading of image rays to pose the corresponding inverse problem. We have suggested a set of numerical algorithms for solving the problem numerically. We have tested these algorithms on a synthetic data example with laterally heterogeneous ve- 


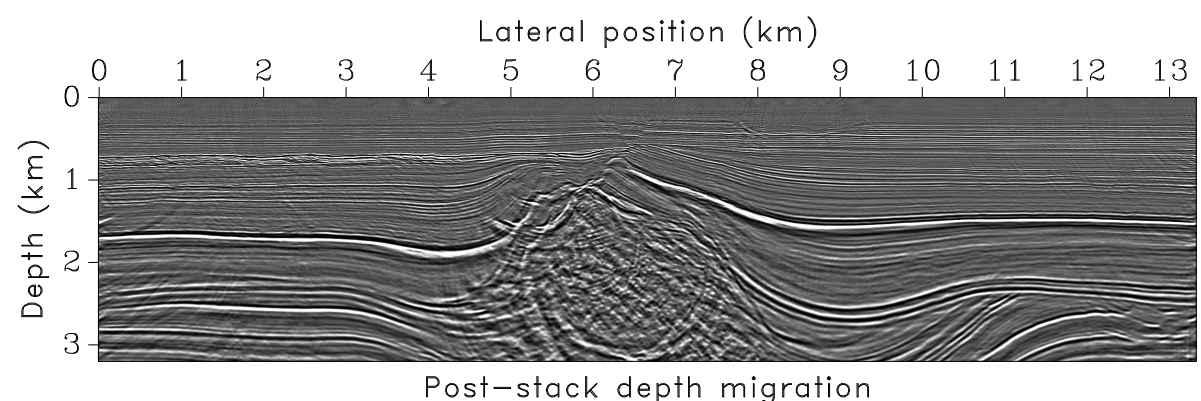

(a)

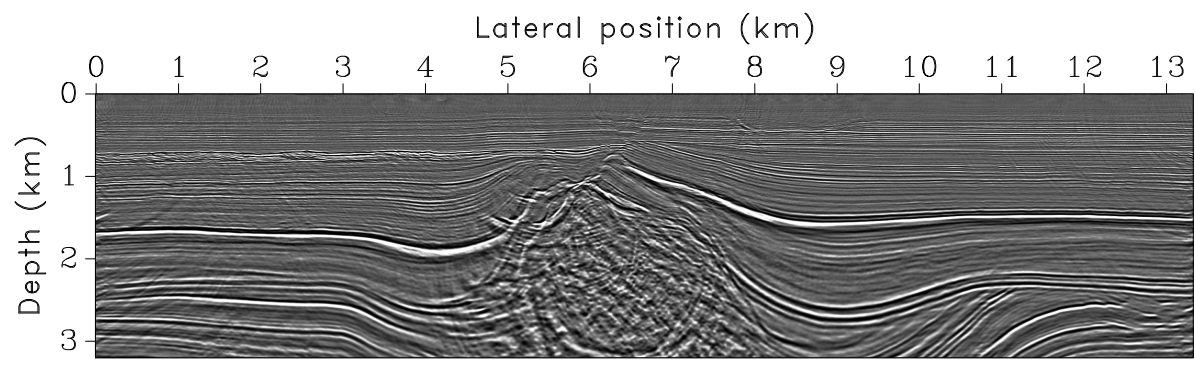

Post-stack depth migration

(b)

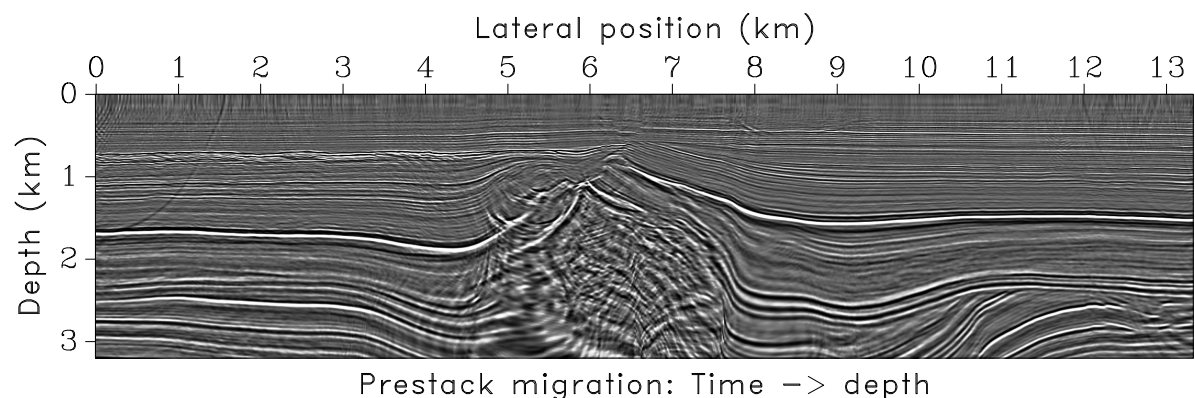

(c)

Figure 5: Migrated images of the field data example. (a) Poststack migration using Dix velocity. (b) Poststack migration using estimated velocity. (c) Prestack time migration converted to depth with our algorithm. 
locity and demonstrated that they produce significantly better results than simple Dix inversion followed by time-to-depth conversion. Moreover, the Dix velocity may qualitatively differ from the output velocity. We have also tested our algorithm on a field data example and validated it by comparing prestack time migration image mapped to depth with post-stack depth migrated images. Our approach is complementary to velocity estimation methods that work directly in the depth domain. Therefore, it can serve as an efficient first step in seismic velocity model building.

\section{REFERENCES}

Bevc, D., J. L. Black, and G. Palacharla, 1995, Plumes: Response of time migration to lateral velocity variation: Geophysics, 60, 1118-1127.

Boyd, J. P., 2001, Chebyshev and Fourier spectral methods, 2nd ed. (revised): Dover Publications.

Cameron, M., S. Fomel, and J. Sethian, 2007, Seismic velocity estimation from time migration: Inverse Problems, 23, 1329-1369.

Červený, V., 2001, Seismic ray method: Cambridge University Press.

Dix, C. H., 1955, Seismic velocities from surface measurements: Geophysics, 20, 68-86.

Fomel, S., 2003, Time-migration velocity analysis by velocity continuation: Geophysics, 68, 1662-1672.

Hatton, L., L. K. Larner, and B. S. Gibson, 1981, Migration of seismic data from inhomogeneous media: Geophysics, 46, 751-767.

Hubral, P., 1977, Time migration - Some ray theoretical aspects: Geophysical Prospecting, 25, 738-745.

Hubral, P., and T. Krey, 1980, Interval velocities from seismic reflection time measurements: Soc. of Expl. Geoph.

Kim, Y. C., W. B. Hurt, L. J. Maher, and P. J. Starich, 1997, Hybrid migration: A cost-effective 3-D depth-imaging technique: Geophysics, 62, 568-576.

Larner, K. L., L. Hatton, B. S. Gibson, and I. S. Hsu, 1981, Depth migration of imaged time sections: Geophysics, 46, 734-750.

Lax, P. D., 1954, Weak solutions of hyperbolic equations and their numerical computation: Communications in Pure and Applied Mathematics, 7, 159-193.

Popov, M. M., 2002, Ray theory and Gaussian beam method for geophysicists: EDUFBA.

Popov, M. M., and I. Pšenčik, 1978, Computation of ray amplitudes in inhomogeneous media with curved interfaces: Studia Geophysica et Geodaetica, 22, 248-258.

Robein, E., 2003, Velocities, time-imaging and depth-imaging in reflection seismics: EAGE.

Sethian, J., 1996, Fast marching level set method for monotonically advancing fronts: Proceedings of the National Academy of Sciences, 93, 1591-1595.

—, 1999, Fast marching methods: SIAM Review, 41, 199-235.

Siliqi, R., and N. Bousquié, 2000, Anelliptic time processing based on a shifted hyperbola approach: 70th Annual International Meeting, Soc. of Expl. Geophys., 
$2245-2248$.

Yilmaz, O., 2001, Seismic data analysis: Soc. of Expl. Geophys.

Yilmaz, O., I. Tanir, and C. Gregory, 2001, A unified 3-D seismic workflow: Geophysics, 66, 1699-1713. 